\title{
Cable and Phase Identification based on Power Line Communication
}

\author{
Hee-Jung Byun ${ }^{1}$ and Su Goog Shon ${ }^{2 *}$ \\ ${ }^{1}$ Department of IT Engineering, The University of Suwon, IT Building, san 2-2 \\ Wau-ri, Bongdam-eup, Hwaseong-si, Korea \\ ${ }^{2}$ Department of IT Engineering, The University of Suwon, IT Building, san 2-2 \\ Wau-ri, Bongdam-eup, Hwaseong-si, Korea \\ Email: ${ }^{1}$ heejungbyun@suwon.ac.kr, ${ }^{2 *}$ sshon@suwon.ac.kr(correspondingauthor)
}

\begin{abstract}
Phase and cable identification is a challenging issue. Power-line communication technology is applied to identify cable and phase of power distribution systems. Power cable circuits have only a limited ability to carry higher frequencies. Typically transformers prevent propagating the higher frequency carrier signal. The proposed method uses the limited propagation ability to identify the cable as well as the phase. A new cable and phase identification system is designed and implemented. The system consists of a transmitter and a receiver with power-line communication module. Some experiments are conducted to verify the theoretical concepts in a commercial building. Also some simulations are done to help and understand the concepts by using Simulink simulator. It can extend the application area of power-line communication.
\end{abstract}

Keywords: Simulink, Cable Identification, Power-line communication, Distribution lines control communication

\section{Introduction}

Electrical power lines are usually classified into high, medium and low voltage lines. High voltage lines form the transmission line network that transports energy at over $100 \mathrm{kV}$ from generating power plants to electrical substations located near demand centers. Medium voltage lines (typically below $50 \mathrm{kV}$ ) and low voltage lines (less than $1 \mathrm{kV}$ ) form the distribution line network, which carries electricity from the transmission system and delivers it to consumers.

Distribution lines branch into multiple circuits to power local pole- or padmounted transformers [1]. Various distribution lines topologies exist and differ from country to country, and also within each country, for example radial and interconnected distribution networks, delta and wye three-phase services, singlephase and split-phase low-voltage services, different earthing systems.

Electrician on site should correctly figure out electrical configurations such as phases and cable connections. The unmistakable identification of cables and phases is an absolute safety related task. When confusion in lines for phases and cables occurs, disproportionate concentrations of loads can easily occur, and this type of disproportionate concentration of loads can cause phase unbalance that can lead to power loss or power failure and, further, equipment failure due to excessive voltage regulation or reduction of useful life, etc. What this would all mean is that it can result in substantial management difficulties such as economic losses, all because of the reduced quality of voltage supplied to the customer. A wrong selection can result in fatal consequences for the operator and cause the loss of supply for the connected customers. Thus, electrician must become able to figure out there in the 
field as to which phase of $\mathrm{A}, \mathrm{B}$ and $\mathrm{C}$ phases the line conductors belong and as to which transformer the line conductors is connected.

Usually in a substation, electric power is provided to end customers by means of A, B, C three phases through three or four conductors. From MV/LV transformer on electric pole or pad, electric power is serviced one or three phases through two, three or four conductors. As electrician approaches toward the end of the distribution lines, the effort to distinguish both an absolute phase value and cable configurations to MV/LV transformer becomes more difficult.

The need to determine phases and cables is increasing. The determination of an absolute phase is difficult when it is needed on site. As such, most of phase distinguishing methods employ methods that distinguish phases based on comparing the known phase value of a substation with the unknown phase value of a local site [2-7]. For the phase determination, GPS technology is utilized to synchronize time at different locations, which is used to measure the phase for electric signals [8].

In addition to the phase identification, the cable identification is also a challenging issue. The determination of cable connectivity is difficult when it is needed on site. The recent cable identification system, newly developed, consists of current impulse generator and receiver. The receiver is connected by a clamp for decoupling the identification signal. The pulse generator generates some special type of pulses and transmits them into the cables being identified [9]. The sent impulse signal causes an electromagnetic field with a defined polarity around the cable which is received with the flex coupler of the receiver, automatically synchronized. The directional clamp in combination with the parameter monitoring by the receiver provides a safe selection regardless of any interference. It means that only one conductor or cable has the correct polarity while all other cables have the opposite polarity. As another method, direct signals are injected on the transmitter and the received signals are analyzed in terms of amplitude-time-phase at receiver [10]. Another method is to communicate coded messages between central device and line device through the distribution networks [11].

Consider the situation that many transformers and distribution lines are deployed along the street as shown in Figure 1. There, an electric technician wants to find out transformer or distribution line to which premise power line cables are connected. It is not easy to figure out because there are too many obstacles such as buildings, trees, hills.

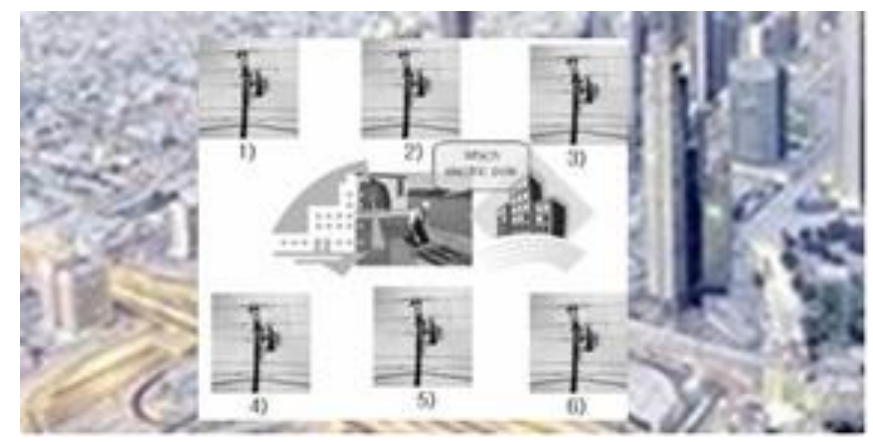

Figure 1. Situation to Require Cable Identification

In this paper, we propose how to find out the cable and phase identification by using power-line communication for the easy, reliable and safe selection of cables. The paper uses some facts that power cable circuits have only a limited ability to carry higher frequencies. A new cable identification system is designed and implemented. The system consists of a transmitter and a receiver with power-line communication module. Some experiments are conducted to verify the theoretical 
concepts. Also some simulations are done to help and understand the proposed system by using Simulink simulator.

\section{Proposed Architecture of Phase and Cable Identification System}

The power network can support communication channel based on power line communication technology. Power line communications have been used at all levels of the power network. A wide range of power-line communication technologies are needed for different applications, ranging from narrowband to broadband over power lines (BPL); on high voltage lines for telemetry, protection and control by utility companies; on medium distribution voltage lines for advanced metering and grid optimization by electricity providers, but also for high-speed Internet distribution; finally, the vast majority of PLC applications are on low distribution voltage lines, both indoor and outdoor (between transformers and buildings), for both low-speed command and control applications and high-speed networking. Advanced metering infrastructure (AMI) systems becomes recently one of applications widely used.

In spite of the growing use of digital communication systems, PLC is still often the most cost effective and reliable solution to cover the operational needs of a power network. The key advantage of PLC is the use of existing electrical lines as communication medium, which provides the major benefit of eliminating considerable costs of installing networking infrastructure, like dedicated cables or antennas. Data is sent on the power lines by superposing a modulated highfrequency carrier signal on the power line voltage, being high, medium or low, AC or DC. The carrier signal is then de-coupled and demodulated at the receiving end to recover the information.

We distinguish today between two classes of PLC systems: narrowband and broadband. Usually, narrowband PLC (NB-PLC) refers to low bandwidth communication, utilizing the frequency band below $500 \mathrm{kHz}$ and providing data rates of tens of kpbs. Broadband PLC (BPL) utilizes a much wider frequency band, typically between $2 \mathrm{MHz}$ and $30 \mathrm{MHz}$, and allows for data rates of hundreds of Mbps.

The power lines were not designed for data transmission and they constitute a challenging environment for communication. The transmission network and even more the distribution network have the characteristics of a very complicated wired network, which vary with time, location and are frequency-dependent. The main factors that affect the communication performance are high signal attenuation and considerable noise. Also, severe electromagnetic compatibility restrictions impose non-negligible challenges to PLC systems. Those are the reasons why most PLC technologies limit themselves to one type of wires (such as premises wiring within a single building). These characteristics can inversely be utilized to identify cables.

Typically power distribution transformers prevent propagating the high frequency signal, which requires multiple technologies to form very large networks [12]. Various data rates and frequencies are used in different situations. Power distribution transformers cause high signal attenuation when PLC devices are located on primary and secondary sides, for example in communication between meters on the low voltage line and a concentrator connected on medium voltage side. Both signal propagation on multiple paths and signal reflection due to impedance mismatches in branching points can generate attenuation distortion of PLC signals. This phenomenon is less observed at low communication frequencies (below $150 \mathrm{kHz}$ ), since the signal wavelength is quite long (over $1 \mathrm{~km}$ ).

Whatever the type of modulation used, a PLC transceiver includes a few basic blocks as shown in Figure 2. Different solutions use various levels of integration of 
these components, from full-digital modems with external discrete components to highly integrated systems-on-chip [13].

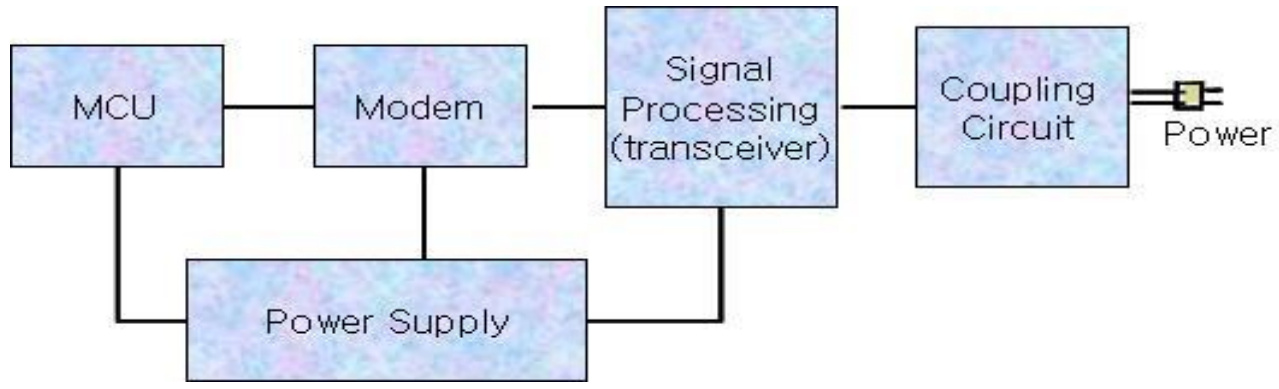

Figure 2. Architecture of PLC transceiver

The core of each transceiver is the modem itself, which usually implements the physical and data link layers, according to the OSI model. Modulationdemodulation, error correction, media access control are among the major functions performed at this level. A MCU unit is responsible for networking, protocol, and application-specific functions. At the other side, transmitted and received signals are processed, in digital and analog form; the main operations include filtering and amplification.

Figure 3 suggests how to identify the particular distribution transformer among three transformers which serve electric power from the secondary side of a distribution transformer to end customers. Power-line communication systems operate by adding a modulated carrier signal to the wiring system. Modulated high frequency carrier signal is transmitted along a power line from a PLC transceiver and is blocked by a transformer which means that the injected signal does not cross over the primary side of the transformer. The proposed system consists of a client and a server. Carrier signals are transferred between client and server via power-line communication. Since the power distribution system was originally intended for transmission of ac power at typical frequencies of 50 or $60 \mathrm{~Hz}$, power cables have only a limited ability to carry higher frequencies $[14,15,16]$. The propagation problem shall be a limiting factor for each type of power-line communications. Especially, the transformer would block the carrier signal cross over the transformer. We expect the modulated carrier signal by the client would not cross over the transformer Tr.2. Even though the signal can cross over the transformer Tr.2, its size would be weak enough not to be communicated via PLC modem.

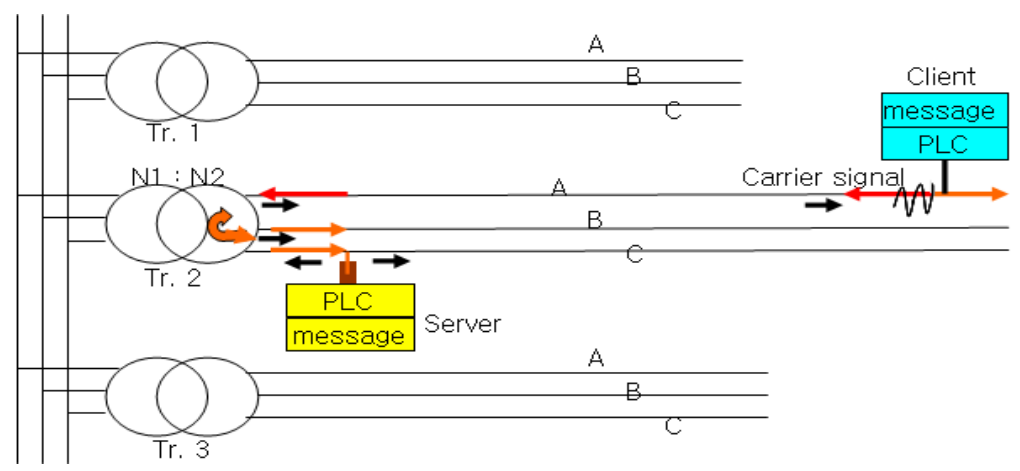

Figure 3. Operation to Identify Transformer

Cable identification methodology can be developed with above characteristics. The injected signal would not be detected from other distribution lines connected 
with Tr.1 or Tr.3. The server unit on distribution lines linked with Tr.2 would respond with the corresponding signal. Also, the responded signal would be received by the client. Then, we would say that the client and the server be connected to the same cable and transformer.

Figure 4 suggests how to figure out cable and phase identification by using more servers with different coded messages. As one client sends out a request message, all the server would respond with different code messages. The client would receive sequentially messages depending on the distance between the client and servers.

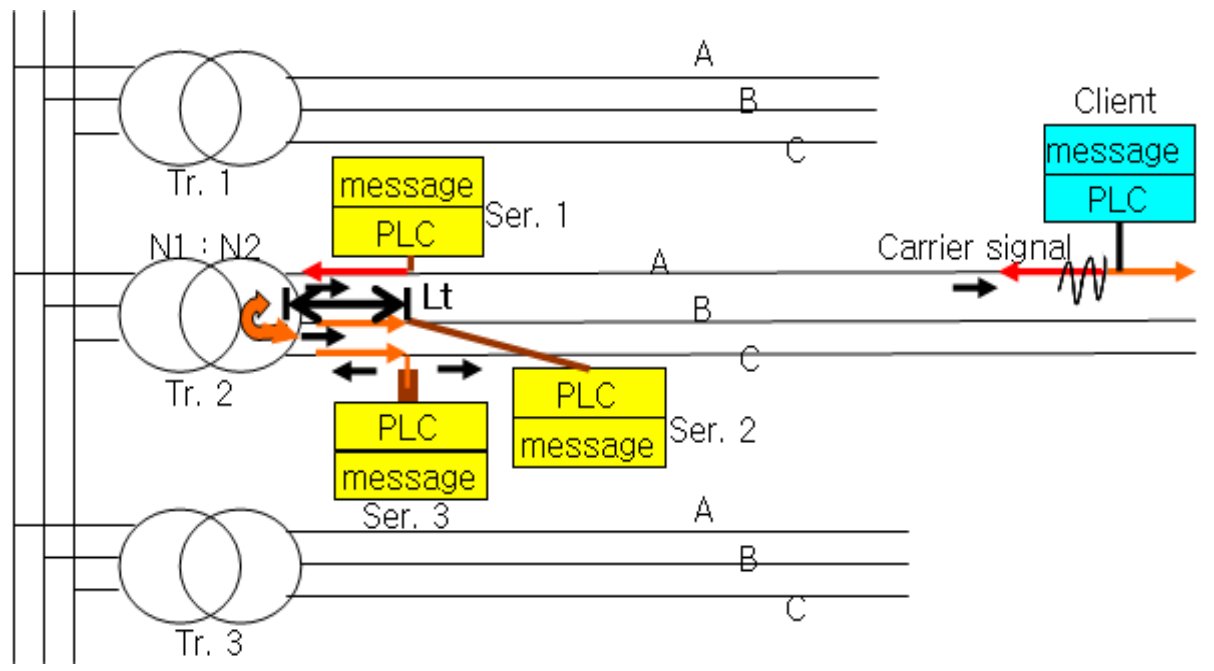

Figure 4. Operation to Identify both Cables and Phases

The distance between the transformer Tr.2 and a server is ' $L t$ '. Also, the distances between the transformer Tr.2 and other servers are 'Lt's. Consider that the client starts send message at 'to' and would receive message from the nearest server 1 at time ' $t 1$ '. The client may receive later other messages from other servers (ser. 2 and ser. 3) at time 't2' ('t3') as shown in Figure 5. Geographically, other servers (ser. 2 and ser. 3 ) are located far way two times of 'Lt'.

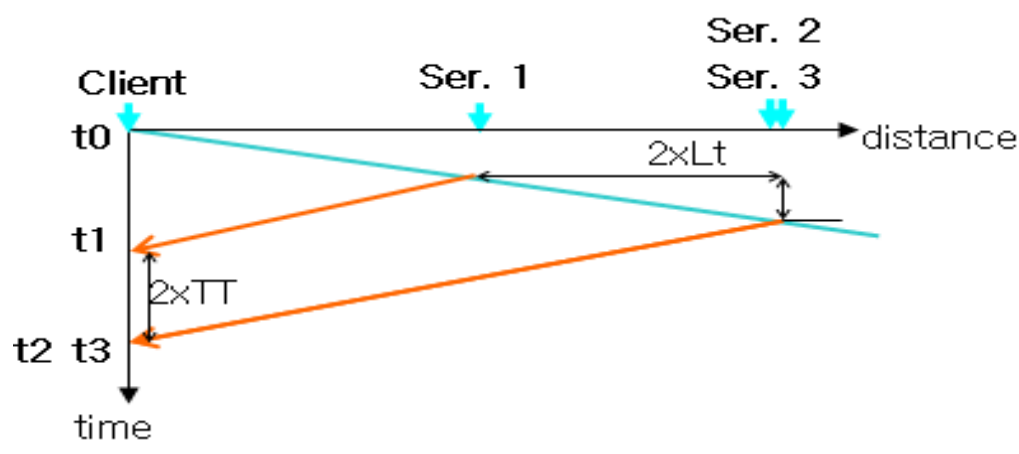

Figure 5. Flow of Operation between the Client and the Servers

The transmission time that the carrier signal transmits over the distance ' $\mathrm{Lt}$ ' becomes ' $\mathrm{TT}$ ' $=\mathrm{Lt} / \mathrm{S}$, where ' $\mathrm{S}$ ' is the propagation speed of carrier signal. Finally, the client would receive different code messages at different times, 't 1 ', ' $\mathrm{t} 2$ ' $(=\mathrm{t} 1+$ $2 \times \mathrm{TT})$, ' $\mathrm{t} 3$ ' (= $\mathrm{t} 1+2 \times \mathrm{TT})$, respectively. If the client could discriminate the messages received different times (' $\mathrm{t} 1$ ', ' $\mathrm{t} 2$ ', or ' $\mathrm{t} 3$ '), phase and cable identification can be possible. If the servers with coded messages are installed on known phase lines, we can tell which phase the client is connected to after discriminating the 
messages. As an example, $\mathrm{Lt}=100 \mathrm{~m}, \mathrm{~S}=3 \times 10^{8} \mathrm{~m} / \mathrm{s}$. Then the round delay time RDT (= $2 \times$ TT) becomes around 700 microseconds. However, Very fast signal processing is required for the phase and cable identification.

Figure 6 shows the flow chart for algorithm to determine the nearest server. The client and the server need to communicate coded messages each other and resolve the messages. Error detection algorithm is also required for reliable identification. The client and server would be implemented with embedded system.

It is required that both the client and servers are ready. The server only responds whenever there is client request. The client starts to send request message. A server may respond corresponding message with server identification number. After analyzing the received message, the client can determine the nearest server which may be directly connected to the client. The nearest server would respond on the client request in the first if possible discriminates RDT precisely.

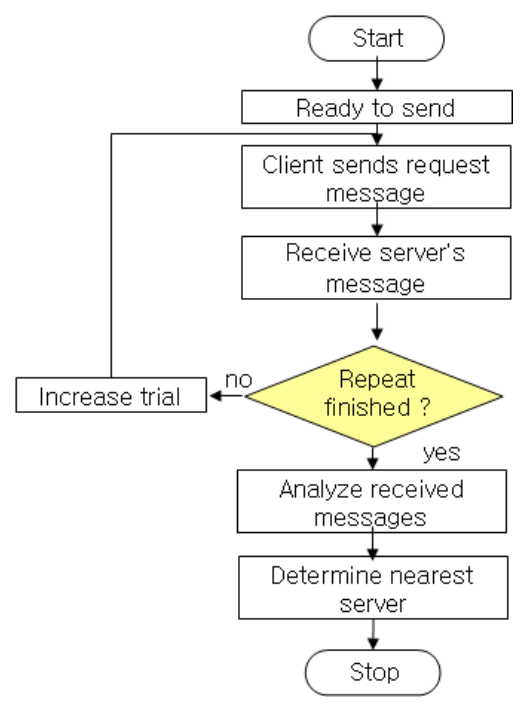

a) client-side algorithm

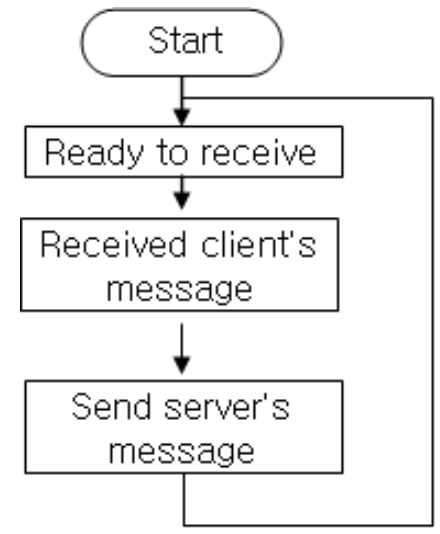

b) server-side algorithm

Figure 6. Flow Chart for Determination of Nearest Server

\section{Simulink Model for 3-phase Distribution Lines}

In order to apply properly the cable identification technology, some analyses about power-line communication should be required for distribution lines. Digital computers can be used to accommodate various methods to do network analysis. Flow calculations, fault current calculations, EMTP analysis, etc., can be 
considered. For analysis, some researches have been done. The two port network is used to represent the low voltage distribution systems and Matlab/Simulink is applied for the analysis of PLC signal attenuation from the secondary side of transformer to the customer locations $[14,15]$. ATP/EMTP is also used to carry out simulation about characteristics of high frequency transformer [17].

In this paper, 3-phase line model is used to simulate signal propagation characteristics for each phase. Traditionally, a 3-phase line was described as a single phase, lumped model by using equal load and balance line conditions, and the analysis that calculates the phase shifts was performed based on the Kirchhoff's voltage law [7].

Figure 7 shows a simplified model of distribution lines that have 3-phase, 4-wire multiple conductors with a multi-grounded neutral. A model will be used to analyze the carrier signal between the two points on a distribution line. To the top of the distribution line model, distribution network model is comprised of 3-phase power sources, circuit breakers, transformers, loads, ground, etc. Distribution network, in particular, can be described with branches going out in radial direction from a substation.

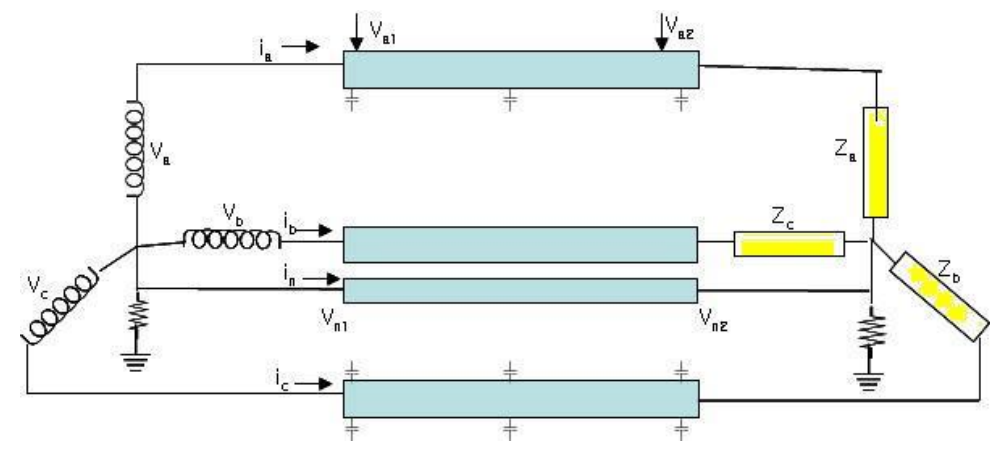

Figure 7. 3-phase 4-wire Grounded wye Line Segment Model

The distribution lines can be modeled as a distributed parameter circuit through the application of, instead of a static model based on the circuit theory of Kirchhoff, the transmitted wave theory. Power signal that goes through a power line moves in accordance with the electromagnetic transmission theory. The power waveform applied to the lines is assumed to be a sinusoidal waveform. The individual phase of a three-phase voltage signal is a $120^{\circ}$ apart and it is of a $60 \mathrm{~Hz}$ frequency. In order to distinguish individual 3 phases, the symbols $A, B$ and $C$ are used. The transmission equation for a power signal is applied to a distribution line.

Applying Kirchhoff's voltage law to the 4-wire grounded neutral line circuit, phase impedance matrix is obtained by Equation (1).

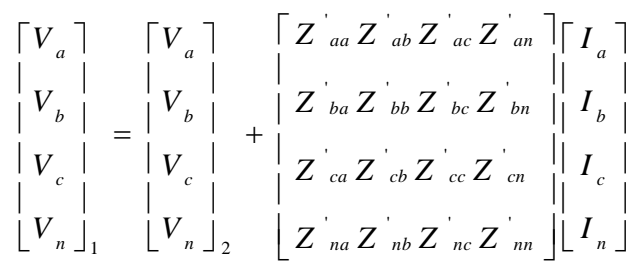

Equation (1) can be reduced $3 \times 3$ phase matrix consisting of the self and mutual equivalent impedances for the three phases. "Kron" reduction method is applied in usual. Finally, Equation (2) for converting line-to-ground phase voltages to sequence voltages is obtained by: 


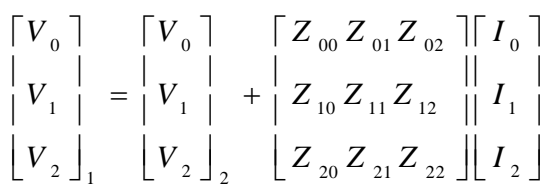

, where the diagonal terms of the matrix are the sequence impedances of the line such that:

$\mathrm{Z}_{00}=$ zero sequence impedance

$\mathrm{Z}_{11}=$ positive sequence impedance

$\mathrm{Z}_{22}=$ negative sequence impedance

For distribution network simulation, MATLAB Simulink software is used [18]. Compared to other software programs, Simulink software offers the advantage of not requiring a compilation process for the source code.

As an example, the Simulink model including several transformers and distribution lines is shown in Figure 8. More details for the Simulink model such as power sources, lines, unbalanced loads, transformers, grounding resister and phase measurement system for phase simulation are simply explained below [8]. In the first, the input source is expressed in terms of power with an internal resistance and inductance. Power capacity that can sufficiently provide power used in the load is arranged. Power is wired as a 3-phase and a supply voltage is sinusoidal. A neutral point is connected to a grounding resistance. The power output is provided to distribution lines through a transformer wired as a wye $(Y)$ or delta $(\Delta)$.

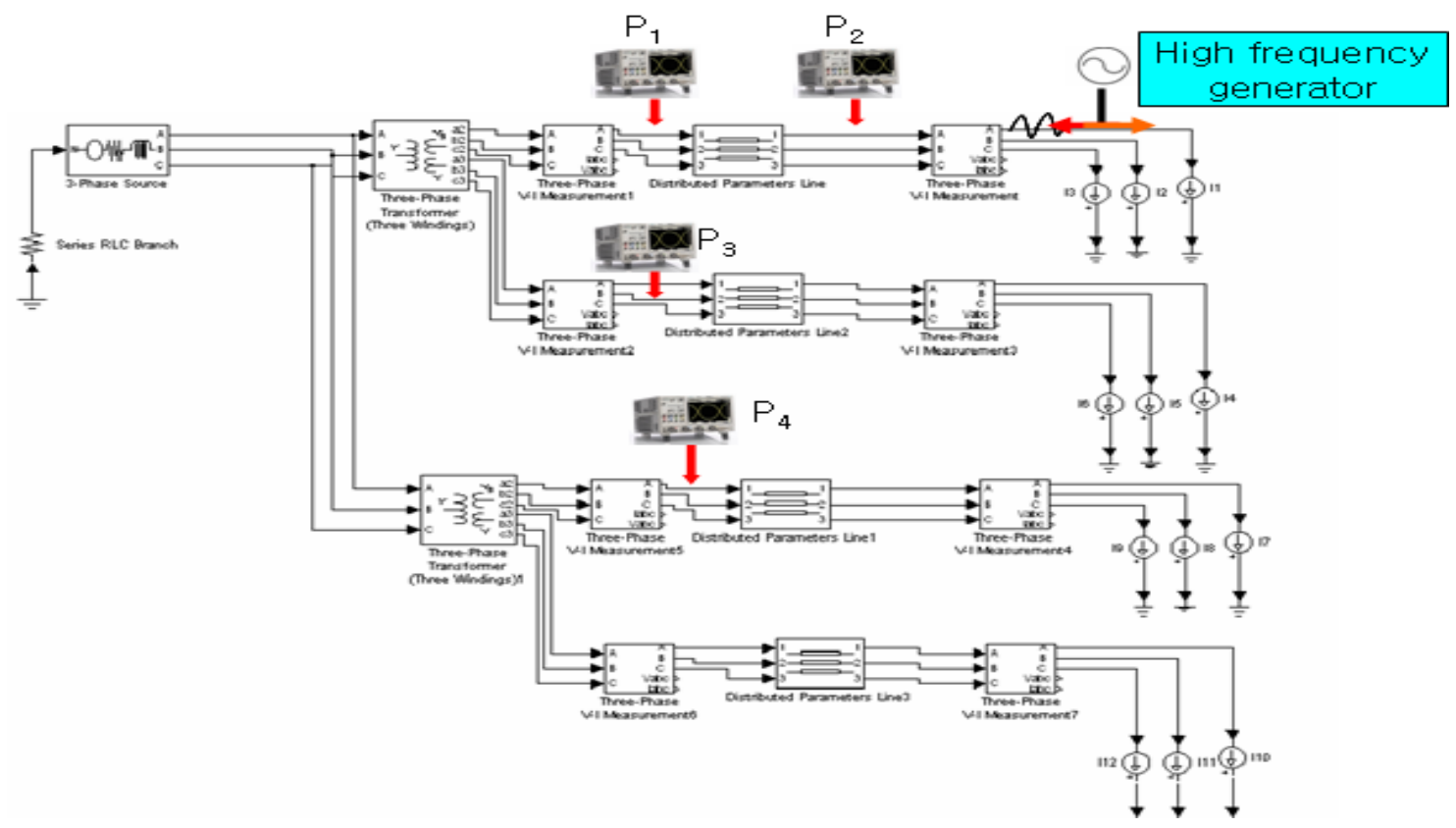

Figure 8. Sample of Simulink Distribution System Model

For the Simulink model of loads, various forms such as constant impedance, a constant current load, or a constant capacity, etc. can be used. A constant impedance model is that linear load that has a series resistance, inductance and capacitance value in a prescribed frequency. The effective and reactive power dissipated in the load has the characteristics of being proportional to square of the applied voltage. Simulink model of a constant current load is useful for allotting any current by lines and is used in this paper.

In modeling a transformer, the voltage ratio of primary and secondary voltages and wiring method can set. Transformer specifications used in simulation comprise 
3-phase transformer with 3 single-phase transformers, and wye $(Y)$ and Delta $(\Delta)$ wiring is possible in the primary and secondary. In this paper, the transformer model that takes into consideration internal loss that is dependent on internal resistance and inductance is used. Recent transformers have very low resistance and voltage drop. Therefore, usually the voltage phase shift across a transformer is calculated as less than $\pm 5^{\circ}$.

Ground is modeled as having only a resistive component. Ground resistance is assumed to be $5 \Omega$, which is a standard value for a $22.9 \mathrm{kV}$ multiple-grounding system [18].

For the distribution lines, parameters are represented as symmetric (zero, positive, negative) components $[7,19,20]$.

\section{Simulation and Implementation Results}

It is to determine the frequencies of power-line communication. Many nations regulate unshielded wired emissions as they have the potential to cause radio transmissions. It is recommended that unlicensed bands or below $500 \mathrm{kHz}$ be used.

By using the sample distribution model shown in Figure 8, the distribution system is analyzed with Simulink when carrier signal of $290 \mathrm{kHz}$ and $300 \mathrm{~mA}$ is injected onto the distribution lines.

With the assumption that electrical signals move at the speed of light is made, propagation time would be required for electrical signals to move a certain distance. There are several factors such as propagation delay, the length of line, the distributed parameters, and load current, etc., to affect the modulation characteristics. The length of ' $\mathrm{Lt}$ ' is used $500 \mathrm{~m}$.

The component values used in the simulation of equation (2) are as follows [21].

Zero Phase Ro $=0.23 \Omega / \mathrm{km}$, Lo $=5.478 \mathrm{mH} / \mathrm{km}$, Co $=0.008 \mu \mathrm{F} / \mathrm{km}$

Positive Phase R1 = $0.17 \Omega / \mathrm{km}, \mathrm{L} 1=1.21 \mathrm{mH} / \mathrm{km}, \mathrm{C} 1=0.00969 \mu \mathrm{F} / \mathrm{km}$

Figures 9, 10, and 11 show voltage waveforms at three different positions ( $\mathrm{P} 2$, P3, and P4). In order to show the characteristics of modulated signal, some larger, exaggerated, carrier signal of $300 \mathrm{~mA}$ larger than real signal is used to. The figures show that modulated signal is gone away at P4 which is located on the other transformer.

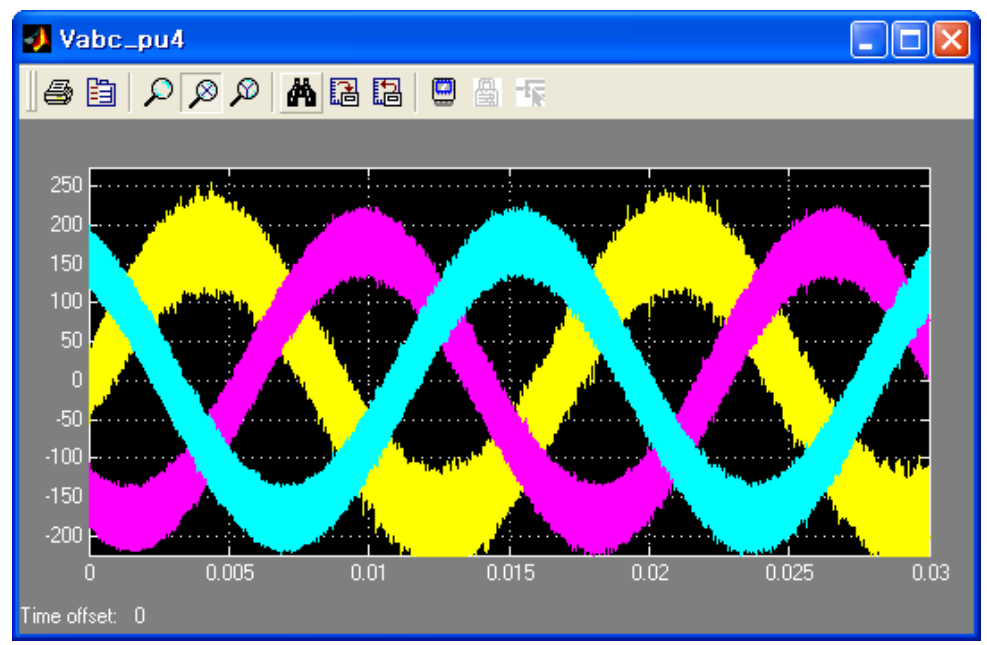

Figure 9. Voltage Waveform at P2 for Carrier Signal of $290 \mathrm{kHz}$ and 300mA 


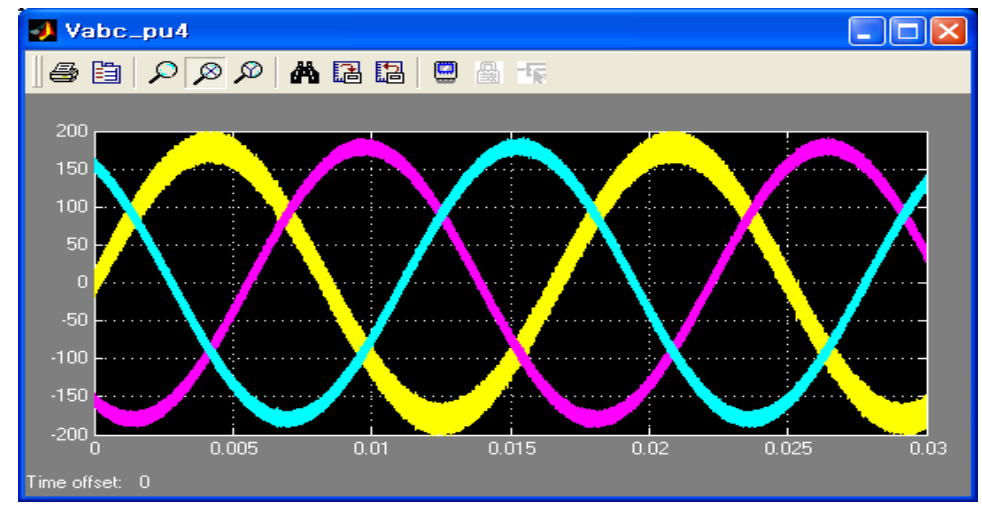

Figure 10. The Secondary-side Low Voltage Waveform at P3 for Carrier Signal of $290 \mathrm{kHz}$ and 300mA

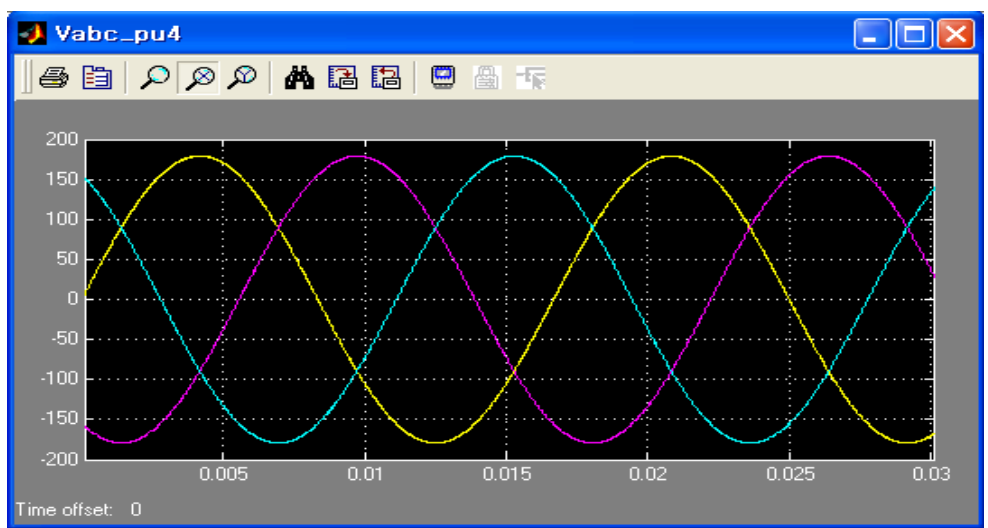

Figure 11. The Secondary-side Low Voltage Waveform at P4 of the Other Transformer Cross One Transformer for Carrier Signal of $290 \mathbf{~ k H z}$ and 300 mA

Figure 12 shows architecture of cable identification systems, which is comprised of a server and a client. Message from client program is transferred into PLC modem through interface. PLC modem converts the message into signal, which is modulated into higher carrier frequency. The modulated signal is transferred into the server. The server side's PLC modem receives the modulated signal and demodulates that into data. Then it is moved into the microprocessor. The server program responds to the client.

The power needs to activate the microprocessor and system. The power source unit is for the power. It is also important to determine the frequencies of power-line communication between broadband PLC (BPLC) and the narrowband PLC (NBPLC). Here the carrier frequencies are $290 \mathrm{KHz}$ and $125 \mathrm{KHz}$ with narrow band modulation of simple MAC. 


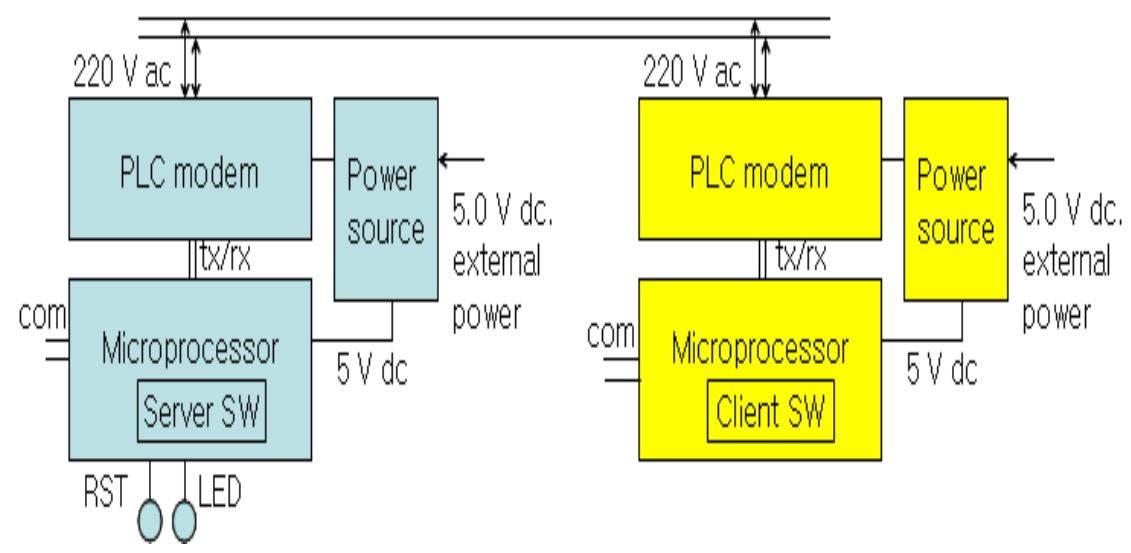

Figure 12. Architecture of Cable Identification Systems

A test system is implemented and tested. The PLC modem chip with carrier frequency of $290 \mathrm{kHz} / 125 \mathrm{kHz}$ and narrowband simple MAC is used. Arduino Uno board is used for microprocessor unit. [22]

Experiments done in a big commercial building show that the PLC carrier signal is transferred into the range of PLC carrier propagation. The high frequency carrier signal does not cross over the secondary side of a transformer.

\section{Conclusions}

For the proper application of the cable and phase identification technology, characteristics of higher frequency carrier propagation are analyzed through several transformers with Simulink. Simulink models and theoretical methods are presented for the analyses. Theoretical simulation results in that the injected carrier signal is hard to cross over the higher level transformer.

In order to verify the idea, the proposed cable identification system is implemented with narrowband power-line communication module with simple MAC and $290 \mathrm{kHz}$ and $125 \mathrm{kHz}$. Experiments show also that the carrier signal is not transferred over the higher level transformer.

For the further research topics, more experiments are considered.

\section{Acknowledgements}

This study was conducted by [GRRC (Gyeonggi Regional Research Center) Suwon 2015-B4, Development of smart emergency response and application systems] as part of projects of GRRC, Gyeonggi Province. The advice received from S. J. Choi of Editech, Ltd. is highly appreciated.

\section{References}

[1] C. D. Scott and R. E. Smalley, J. Nanosci. Nanotechnol, vol. 3, no. 75, (2003)

[2] J. McDonald, "Electric Power Substations Engineering," 2nd, CRC Press, (2007).

[3] M. Bouvrette, "Telephasing method and system for remotely identifying unknown phases of transmission or distribution lines within an electrical network," US Patent 4626622, (1986).

[4] L. A. Pomatto, "Apparatus and method for identifying the phase of a three phase power line at a remote location", US Patent 5510700, (1996).

[5] K.E. Martin, "IEEE Standard for Synchrophasers for Power Systems", IEEE Transactions on Power Delivery, vol.13, no.1, (1998), pp73-77.

[6] G. H. Piesinger, "Apparatus and method for identifying cable phase in a three-phase power distribution network", US Patent 7,031,859, (2006).

[7] W. H. Kersting, "Distribution System Modeling and Analysis", CRC Press, (2002). 
[8] H. J. Byun and S. G. Shon, "Phase Shift Analysis and Phase Identification for Distribution System with 3-Phase Unbalanced Constant Current Loads”, J. Electr. Eng. Technol, vol. 8, no.4, pp. 742-749, (2013).

[9] http://www.sebakmt.com/products/cable-identifier.html

[10] www.powerpoint-engineering.com BAUR KSG 100 cable identifier

[11] F. L. Bertotti, M. S. Hara, and P. J. Abatti, "A simple method to measure phase difference between sinusoidal signals", Rev. Sci. Instrum, vol. 81, no. 115106, (2010).

[12] C. Andrieu, E. Dauphant, and D. Boss, "A Frequency-Dependent Model For a MV/LV Transformer, IPST '99- International Conference on Power Systems Transients, (1999).

[13] C. Ferreira, L. Lampe, J. Newbury, T.G. Swart, "Power Line Communications: Theory and Applications for Narrowband and Broadband Communications over Power Lines", John Wiley \& Sons, (2010).

[14] C. S. Chen, T. T. Ku, C. H. Lin, "Design of PLC based identifier to support transformer load management in Taipower", IEEE Transactions on Industry Applications, vol. 46, no. 3, (2010), pp.10721077.

[15] T. T. Ku, C. S. Chen, C. H. Lin, and M. S. Kang, "Identification of customers Served by Distribution Transformer using Power Line Carrier Technology", The $4^{\text {th }}$ IEEE Conference on Industrial Electronics and Applications, (2009), pp 3476-3481.

[16] A. Cataliontti, G. Tine, "On the MV Power Line Communication System in the Case of Line to Line Transmission”, 2009 XIX IMEKO World Congress, (2009), pp 892-895.

[17] T. Tran-Ahn, P. Auriol, and T. Tran-Quoc, "High frequency power transformer modeling for Power Line Communication applications", 2006 IEEE PES Power Systems Conference and Exposition PSCE, (2006), pp 1069 -1074.

[18] "IEEE Recommended Practice for Grounding of Industrial and Commercial Power Systems", IEEE Std142, (1991).

[19] J.R. Carson, "Wave propagation in overhead wires with ground return", Bell System Technical Journal, vol. $5,(1926)$.

[20] G. Kron, "Tensorial analysis of integrated transmission systems", AIEE Transactions, vol. 71, no. 1952.

[21] W. G. Zhang, "Application of Wavelet Packet Analysis in Phase-to-Ground Fault Detection of Distribution Power System", The International Conference on Electrical Engineering, (2009).

[22] H. J. Byun, C. Feng, S. Shon, "Implementation of a WLAN DMX Server based on NDIS WLAN Miniport Driver", International Journal of Smart Home, (2013), vol. 7, no. 4, pp. 361.

\section{Authors}

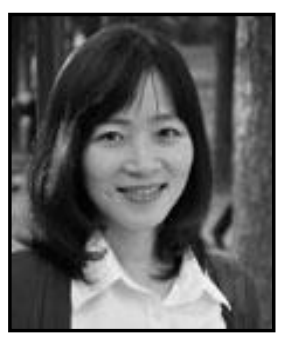

Heejung Byun, she received the B.S degree from Soongsil University, Korea, in 1999, the M.S. degree from Korea Advanced Institute of Science and Technology (KAIST), Korea, in 2001, and the Ph.D. degree from KAIST in 2005. She was a senior researcher in Samsung Electronics, Ltd. from 2007 to 2010. She is currently an assistant professor with the Department of Information and Telecommunications Engineering, Suwon University, Korea. Her research interests include network protocol, network modeling, controller design, and performance analysis.

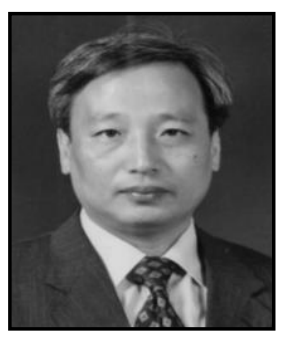

Sugoog Shon, (Corresponding author) he received his B.S. (1982) degree in Electrical Engineering from Seoul National University, his M.S. (1984) degree in Electrical Engineering from Seoul National University, and his Ph.D. (1996) degree in Electrical and Computer Engineering from the University of Texas at Austin. He is a professor in the Department of Information and Telecommunication at the University of Suwon in Korea. His research interests include computer and embedded system, robot, network protocol, network simulation, and network programming. 